Experimental demonstration of phase resonances in metallic compound gratings with subwavelength slits in the millimeter wave regime

Miguel Navarro-Cía, Diana C. Skigin’, Miguel Beruete, and Mario Sorolla

Citation: Appl. Phys. Lett. 94, 091107 (2009); doi: 10.1063/1.3086892

View online: http://dx.doi.org/10.1063/1.3086892

View Table of Contents: http://aip.scitation.org/toc/apl/94/9

Published by the American Institute of Physics

AIP $\mid$ Applied Physics Letters

If your article has the

Save your money for your research.

It's now FREE to publish with us -

no page, color or publication charges apply. 


\title{
Experimental demonstration of phase resonances in metallic compound gratings with subwavelength slits in the millimeter wave regime
}

\author{
Miguel Navarro-Cía, ${ }^{1}$ Diana C. Skigin, ${ }^{2, a)}$ Miguel Beruete, ${ }^{1}$ and Mario Sorolla ${ }^{1}$ \\ ${ }^{1}$ Millimeter and Terahertz Waves Laboratory, Universidad Pública de Navarra, Campus Arrosadía, \\ 31006 Pamplona, Spain \\ ${ }^{2}$ Grupo de Electromagnetismo Aplicado, Departamento de Física, Facultad de Ciencias Exactas y \\ Naturales, Universidad de Buenos Aires, Ciudad Universitaria, Pabellón I, C1428EHA Buenos Aires, \\ Argentina
}

(Received 31 October 2008; accepted 1 February 2009; published online 4 March 2009)

\begin{abstract}
We provide experimental evidence of phase resonances in metallic periodic structures in which each period comprises several subwavelength slits of the same width. We have analyzed and measured the response of these structures in the millimeter wave regime and show that phase resonances are characterized by a remarkable minimum in the transmission response, as predicted by numerical calculations. We compare experimental with numerical results, obtaining a very good agreement between them. This experimental confirmation encourages research in compound structures and their multiple potential applications, such as frequency selective surfaces. (C) 2009 American Institute of Physics. [DOI: 10.1063/1.3086892]
\end{abstract}

It is well known that the transmitted response of a regular array of slits presents a series of Fabry-Pérot-like resonances, which appear as peaks in the transmittance for certain wavelengths. ${ }^{1}$ This transmission enhancement corresponds to waveguide resonances within each individual slit, and their location is related to the thickness of the structure. Consequently, the resonant wavelengths are nearly the same for structures with one, several, or many slits. Research on one-dimensional metallic structures has considerably increased in the last decade due to the enhanced transmission phenomenon first reported in a metallic plate with holes. ${ }^{2}$ Theoretical $^{1,3,4}$ as well as experimental ${ }^{5,6}$ research has been carried out in connection with slit structures and their capability of producing enhanced transmission.

On the other hand, not so much attention has been paid to the study of dual-period structures. The first studies of the response characteristics of compound gratings were carried out for reflection structures, ${ }^{7-9}$ and later on the same phenomenon was found in transmission structures. ${ }^{10-13}$ It has been shown that transmission metallic gratings comprising several subwavelength slits within each unit cell exhibit phase resonances, i.e., resonances that are characterized by a phase reversal of the magnetic field in adjacent slits within each period. ${ }^{10,11}$ Such resonances only take place under $p$-polarized incidence (electric field in the plane of incidence), and they are only permitted when the period is formed by several slits or cavities. Phase resonances appear in the transmitted response as pronounced dips within a waveguide-mode resonance peak. With the increase in the number of slits in each period, more degrees of freedom are introduced, that allow for more possible near field configurations in which a phase reversal between adjacent slits is found.

Only a few recent works reported experimental demonstrations of phase resonances in slit structures. Hibbins et $a l .{ }^{12}$ measured the transmissivity of an air-filled compound grating comprised of two narrow slits flanking a wider cen-

${ }^{a)}$ Electronic mail: dcs@df.uba.ar tral slit under microwave radiation which is characteristic of phase resonances and Ma et al. ${ }^{13}$ reported measurements for periodic structures with unit cells consisting of two or three slits of different widths.

In this work we report experimental demonstration of phase resonances in compound structures comprising several slits of the same width, in the millimeter wave regime. Samples of one to three equal slits per period have been fabricated, and their transmittance and reflectance have been measured and compared with the results obtained by numerical calculations. Our work differs from Ref. 12 in several aspects. Especially, we include an analysis on the excitation of phase resonances in a two-slit-per-period structure, taking into account fabrication errors and slightly off-normal illumination effects.

In Fig. 1 we schematize a compound transmission grating with three slits within the period $(J=3)$. The period of the structure is $d$ and the slit width and height are $c$ and $h$, respectively. The structure is illuminated by a linearly polarized Gaussian beam of wavelength $\lambda$. Several samples with one to three slits per period were fabricated by wire-cut electrical discharge machining in thick aluminum wafers, as shown in Fig. 2. All the aluminum wafers have a diameter of $62.4 \mathrm{~mm}$, and the geometrical parameters are $d=2.8 \mathrm{~mm}$, $c=a=0.4 \mathrm{~mm}$, and $h=2.5 \mathrm{~mm}$.
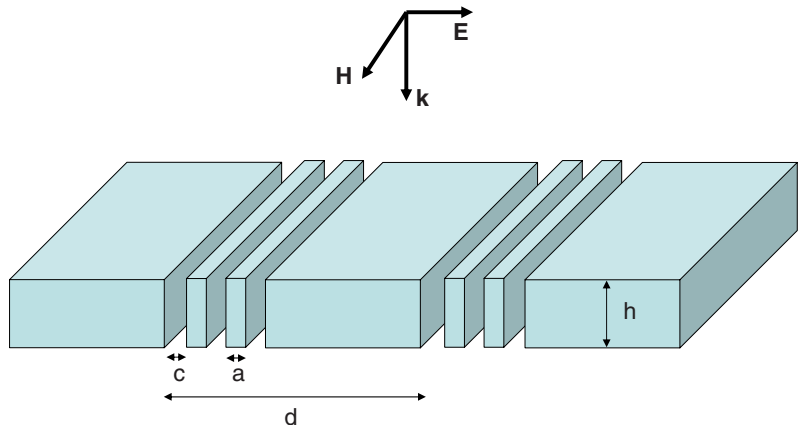

FIG. 1. (Color online) Scheme of the compound grating. 


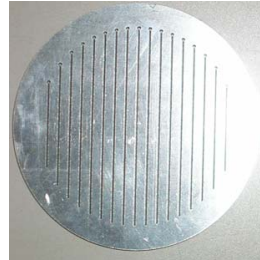

(a)

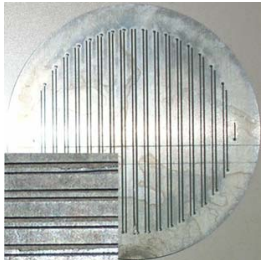

(b)

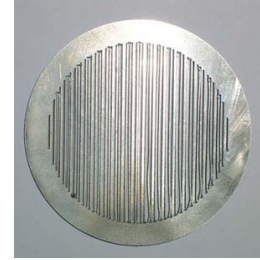

(c)
FIG. 2. (Color online) Fabricated structures: (a) $J=1$ (simple grating); (b) $J=2$; (c) $J=3$.

The measurements of the transmitted intensity were performed with an $\mathrm{ABmm}^{\mathrm{TM}}$ quasioptical (QO) vector network analyzer which can operate from $40 \mathrm{GHz}$ up to $260 \mathrm{GHz}$. This instrument is based on a solid state multiplier that generates the millimeter-submillimeter wave frequencies which are detected by harmonic mixer heterodyne downconversion. The QO setup consists of a corrugated horn antenna that generates a very well linearly polarized Gaussian beam which, after two ellipsoidal mirrors, is focused over the sample under test with a beamwaist of $28 \mathrm{~mm}$. There, the transmitted and reflected beams are obtained. The transmitted beam passes through another pair of identical mirrors and reaches the receiver antenna. This antenna is another corrugated horn which is very sensitive to polarization and well matched to the Gaussian beam. ${ }^{14}$ It has to be noted that the first experimental confirmation of extraordinary transmission in subwavelength hole arrays in the Fraunhofer region and in a slit with grooves structure in the Fresnel region, in the millimeter wave regime-where metals are nearly perfect conductors-was done with this instrument. ${ }^{15,16}$

Experimental reflection and transmission measurements were performed for the three samples with $J=1$ (simple grating), $J=2$, and $J=3$. The results obtained for both polarization modes under normal incidence are compared with numerical simulations in Fig. 3. Two methods have been used to validate the numerical results. From one hand, the classical modal method, ${ }^{17}$ which has already been used to model compound gratings formed by grooves or slits. ${ }^{7,8,10,11}$ On the other hand, CST Microwave Studio ${ }^{\mathrm{TM}}$, a completely numerical approach based upon finite integration time domain method, has been used to validate the results.

In Fig. 3 we show curves of transmitted and reflected intensities as a function of the wavelength. In the frequency range considered, the slits have subwavelength width, and then no transmission is found for $s$ polarization, as expected due to the cutoff frequency for propagating modes. Therefore, all the power is reflected by the structure, regardless of its type (simple or compound).

The picture is different for $p$ polarization, where no cutoff frequency exists and there is at least one propagating mode within the slits even for subwavelength widths. In this case, several peaks in the transmitted response are expected for $p$-polarized incidence, at wavelengths corresponding to the waveguide-mode resonances of each single slit, ${ }^{1,3}$ also called Fabry-Pérot resonances. For ideal slits on a perfectly conducting structure, these resonances are expected to occur at $\lambda=2 h / n$, where $n$ is a positive integer. Then, for the pa-

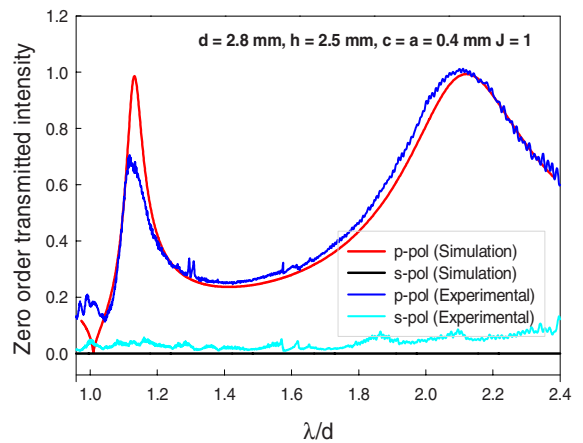

(a)

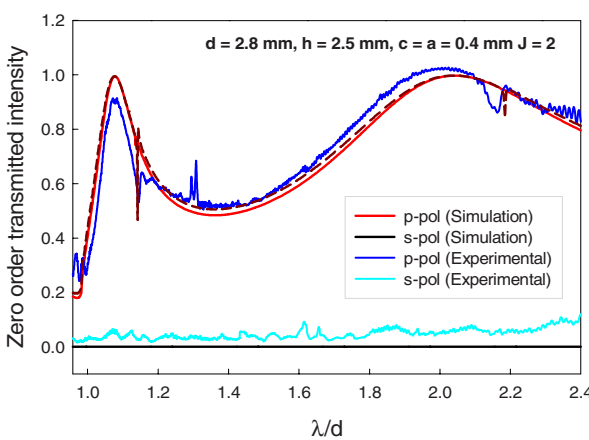

(c)

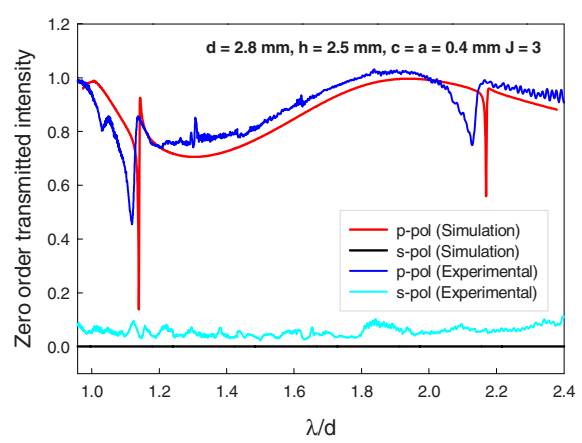

(e)

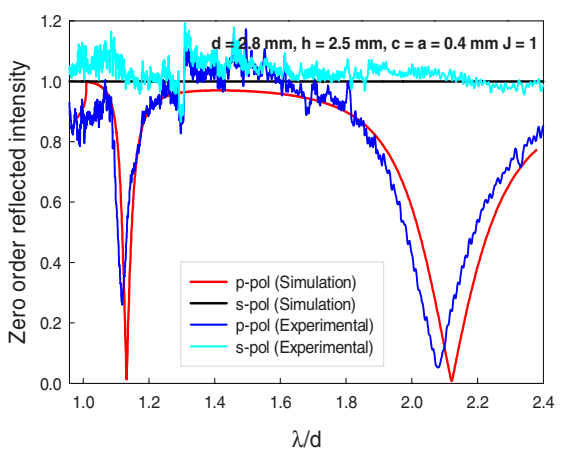

(b)

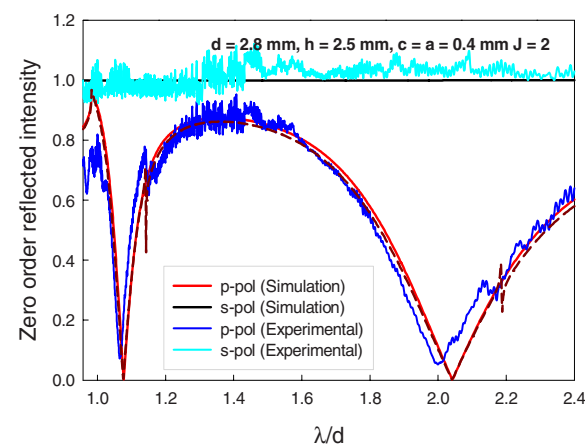

(d)

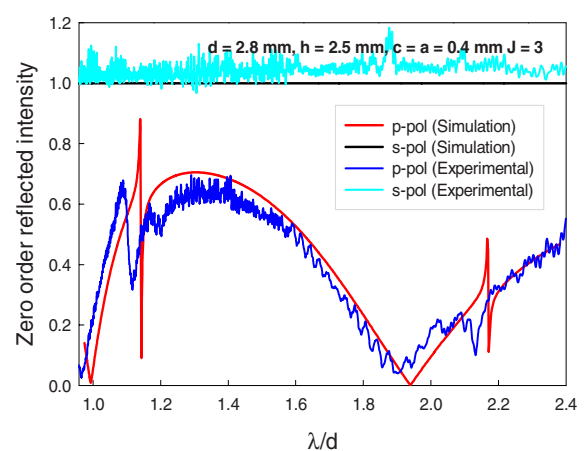

(f)

FIG. 3. (Color online) Reflection and transmission for both polarization modes: measurements and simulations. The parameters of the structures are $d=2.8 \mathrm{~mm}, c=a=0.4 \mathrm{~mm}$, and $h=2.5 \mathrm{~mm}$. (a) Transmission for $J=1$; (b) reflection for $J=1$; (c) transmission for $J=2$; (d) reflection for $J=2$; (e) transmission for $J=3$; (f) reflection for $J=3$. The dashed curves in panels (c) and (d) correspond to numerical simulations for a structure with two slits but with $10 \%$ difference in their widths: $c_{1}=0.4 \mathrm{~mm}$ and $c_{2}=0.44 \mathrm{~mm}$. 
rameters of the fabricated samples, the first two resonances are expected at $\lambda / d \approx 1.8$ and 0.9 , and these values are shared among the three samples since they do not depend on the number of slits per period but only on the thickness-towavelength ratio of each single slit.

For the simple grating (one slit per period, $J=1$ ), two peaks are observed in the transmitted response, which are associated with waveguide-mode resonances [Fig. 3(a)]. These peaks are found at $\lambda / d \approx 2.11$ and 1.13 , i.e., are shifted from the ideal values. This difference comes essentially from the width-to-depth ratio of the slits. As it was shown by Takakura, ${ }^{18}$ there is an increase in the resonant Fabry-Pérot wavelengths even for narrow slits on a perfect conductor. Experimental and numerical results agree very well in this case.

The same two peaks that appear in the $J=1$ curve are present in the $J=2$ and $J=3$ curves [Figs. 3(c) and 3(e)], which correspond to compound structures with two and three slits per period, respectively. It can be observed that as $J$ increases, the peaks are widened and slightly shifted (notice that for $J=3$ only the right part of the first peak remains within the frequency range considered). However, for $J=2$ and $J=3$ a dip is observed within each transmission peak in the experimental curves, which is in good agreement with the numerical predictions for $J=3$ [Fig. 3(e)] but not for $J=2[$ Fig. 3(c)]. These narrow dips are associated with the excitation of phase resonances in compound structures. The fields in all slits of a simple perfectly periodic grating are essentially equal due to the pseudoperiodic condition. However, when slits are added to the period (compound grating), new degrees of freedom open up and the distribution of field phases in the different slits within each period can have different configurations. Under normal incidence, the possible phase configurations must be symmetrical. For instance, for three slits in the period, there are only two possible configurations: (i) all the slits have equal phase $(+++)$ and (ii) the external slits have equal phase, different from the central one. In particular, when the phases in adjacent slits are opposite to each other, $\pi$ resonances can be excited, ${ }^{10}$ and this is the case of the dips at $\lambda / d \approx 1.1$ and 2.1 in Fig. 3(e). For structures with one or two slits in the period under normal incidence, different phases in adjacent slits cannot occur, and therefore no phase resonances should be expected. ${ }^{10}$ However, if the incidence is no longer normal, the symmetry is broken and different phases could be found in adjacent slits, which eventually may generate a phase resonance. ${ }^{11}$ This can partly explain why the measurements and the theory do not agree for $J=2$. Since the horn antennas do not transmit a perfectly planar beam, there are non-normal components that produce a phase resonance even under normal illumination, and this is observed as a very weak feature in the transmitted response. This effect was also observed in Ref. 12 .

The disagreement between the experiment and the simulation in the $J=2$ case can also be attributed to imperfections of the sample, as shown in the inset of Fig. 2(b). It is important to remark that in this case, the limits of this fabrication technology have been reached. The different widths of the slits generate an asymmetry in the sample, which in turn produces the excitation of a phase resonance, as predicted for $J=2$ under oblique illumination $^{11}$ or that observed in Ref. 13 for two nonequal slits. To visualize this effect, we added in Figs. 3(c) and 3(d) a dashed curve which corresponds to the simulation for a structure with two slits with $10 \%$ difference in their widths: $c_{1}=0.4 \mathrm{~mm}$ and $c_{2}=0.44 \mathrm{~mm}$. It can be noticed that the phase resonance minimum is already present in this case (unlike the equal-width slits case) as observed in the experimental data, and this confirms that an error in the sample fabrication can lead to weak phase resonance dips in the transmittance. In both samples $(J=2$ and $J=3)$, the lower quality of the experimental dips compared with the numerical ones can also be attributed to imperfections of the sample, as well as to the finite size of the illuminated area. As expected, for $s$ polarization no resonances are present.

In conclusion, we have experimentally demonstrated the existence of phase resonances in metallic periodic structures in which each period comprises several identical subwavelength slits. Aluminum samples with one to three slits per period have been fabricated and their reflectance and transmittance have been measured. We have compared measured and simulated results in the millimeter wave regime and found a very good agreement between them. The transmittance curves confirm that phase resonances are characterized by a remarkable minimum within the waveguide resonance peaks in the transmission response, as predicted by numerical calculations. We have shown that a slit width of roughly $\lambda / 10$ is sufficiently subwavelength to excite phase resonances. This is an important fact if we think of applying this property for the design of selective devices and also to extend the applicability of this phenomenon to optical wavelengths, where subwavelength slits mean nanosized features.

D.S. gratefully acknowledges partial support from Universidad de Buenos Aires (UBA), and Agencia Nacional de Promoción Científica y Tecnológica (ANPCYT-BID Grant No. 1728/OC-AR06-01785). This work was also supported by Consolider "Engineering Metamaterials" Grant No. CSD2008-00066.

${ }^{1}$ J. A. Porto, F. J. García-Vidal, and J. B. Pendry, Phys. Rev. Lett. 83, 2845 (1999).

${ }^{2}$ T. W. Ebbesen, H. J. Lezec, H. F. Ghaemi, T. Thio, and P. A. Wolff, Nature (London) 391, 667 (1998).

${ }^{3}$ F. J. García-Vidal and L. Martín-Moreno, Phys. Rev. B 66, 155412 (2002).

${ }^{4}$ A. Benabbas, V. Halté, and J.-Y. Bigot, Opt. Express 13, 8730 (2005).

${ }^{5}$ J. W. Lee, M. A. Seo, D. S. Kim, S. C. Jeoung, Ch. Lienau, J. H. Kang, and Q.-H. Park, Appl. Phys. Lett. 88, 071114 (2006).

${ }^{6}$ H. Cao and A. Nahata, Opt. Express 12, 1004 (2004).

${ }^{7}$ A. N. Fantino, S. I. Grosz, and D. C. Skigin, Phys. Rev. E 64, 016605 (2001).

${ }^{8}$ D. C. Skigin, A. N. Fantino, and S. I. Grosz, J. Opt. A, Pure Appl. Opt. 5, S129 (2003)

${ }^{9}$ J. Le Perchec, P. Quémerais, A. Barbara, and T. López-Ríos, Phys. Rev. Lett. 97, 036405 (2006).

${ }^{10}$ D. C. Skigin and R. A. Depine, Phys. Rev. Lett. 95, 217402 (2005).

${ }^{11}$ D. C. Skigin and R. A. Depine, Phys. Rev. E 74, 046606 (2006).

${ }^{12}$ A. P. Hibbins, I. R. Hooper, M. J. Lockyear, and J. R. Sambles, Phys. Rev. Lett. 96, 257402 (2006).

${ }^{13}$ Y. G. Ma, X. S. Rao, G. F. Zhang, and C. K. Ong, Phys. Rev. E 76, 031801 (2007).

${ }^{14}$ C. Dahl, P. Goy, and J. P. Kotthaus, in Millimeter and Submillimeter Wave Spectroscopy of Solids, Topics in Applied Physics Vol. 74, edited by G. Gruener (Springer, Berlin, 1998), pp. 221-280.

${ }^{15}$ M. Beruete, M. Sorolla, I. Campillo, J. S. Dolado, L. Martín-Moreno, J Bravo-Abad, and F. J. García-Vidal, Opt. Lett. 29, 2500 (2004).

${ }^{16}$ M. Beruete, M. Sorolla, I. Campillo, and J. S. Dolado, IEEE Microw. Wirel. Compon. Lett. 15, 286 (2005).

${ }^{17}$ J. R. Andrewartha, J. R. Fox, and I. J. Wilson, Opt. Acta 26, 69 (1977).

${ }^{18}$ Y. Takakura, Phys. Rev. Lett. 86, 5601 (2001). 